\title{
Esofagectomía transhiatal por vía abierta y vía laparoscópica para el cáncer de esófago: análisis de los márgenes de resección y ganglios linfáticos
}

Víctor Valentía, Rally Fares ${ }^{b}$, Neal Reynolds ${ }^{b}$, Patricia Cohen $^{c}$, Nick Theodoro $^{c}$ y Alberto Martínez-lsla ${ }^{c}$

aDepartamento de Cirugía General. Clínica Universitaria de Navarra. Pamplona. Navarra. España.

' Imperial College of London. Londres. Reino Unido.

'West London Upper Gastrointestinal Cancer Center. Londres. Reino Unido.

\section{Resumen}

Introducción. El tratamiento quirúrgico de cáncer de esófago está asociado a alta morbilidad y mortalidad. La cirugía mínimamente invasiva se ha propuesto como una alternativa para intentar reducir estas complicaciones; sin embargo, no hay en la actualidad muchos estudios que valoren la validez oncológica de esta cirugía. El propósito de este trabajo es, de manera preliminar, auditar los resultados en nuestra experiencia de ambas técnicas quirúrgicas centrando la atención especialmente en los aspectos oncopatológicos (márgenes de resección y ganglios linfáticos).

Material y método. Entre abril de 2003 y febrero de 2007 , se intervino a 40 pacientes diagnosticados de cáncer de esófago distal en el Hospital Charing Cross de Londres, 24 por vía abierta y 16 por vía laparoscópica, de acuerdo con el cirujano responsable. EI $50 \%$ de ellos recibieron tratamiento quimioterápico neoadyuvante. Ambos grupos fueron homogéneos para edad, sexo, ASA, estadio tumoral y localización tumoral. En todos los casos se recogió de modo prospectivo en una base de datos el estadio tumoral patológico (TNM), margen distal tumoral, margen proximal tumoral, margen circunferencial tumoral y número de ganglios linfáticos resecados.

Resultados. El número de ganglios linfáticos resecados fue similar en ambos grupos (19 para la vía abierta y 18 para la laparoscópica). El margen distal tumoral medio para el grupo tratado por vía abierta fue de $4,9 \mathrm{~cm}$ frente a 4,3 en el grupo tratado con laparoscopia $(p=0,578)$. El margen proximal tumoral

Correspondencia: Dr. V. Valentí Azcárate.

Clínica Universitaria de Navarra. Universidad de Navarra.

Avda. Pío XII, 36. 31080 Pamplona. Navarra. España.

Correo electrónico: vvalenti@unav.es

Manuscrito recibido el 14-5-2007 y aceptado el 17-9-2007. medio para el grupo tratado por vía abierta fue de 8,4 $\mathrm{cm}$ frente a $4,6 \mathrm{~cm}$ en el grupo por laparoscopia $(p=$ $0,004)$ y el margen circunferencial tumoral fue positivo en $11(45 \%)$ pacientes del grupo de vía abierta frente a $5(33 \%)$ pacientes del grupo laparoscópico ( $p$ $=0,519$ ).

Conclusiones. La cirugía laparoscópica para el cáncer de esófago, en nuestra experiencia, parece mostrar resultados iniciales similares a los ofrecidos por vía abierta en cuanto a número de ganglios linfáticos resecados y márgenes de resección.

Palabras clave: Cáncer de esófago. Cirugía mínimamente invasiva. Esofagectomía transhiatal.

\section{OPEN AND LAPAROSCOPIC TRANSHIATAL OESOPHAGECTOMY FOR CANCER OF THE OESOPHAGUS: ANALYSIS OF RESECTION MARGINS AND LYMPH NODES}

Introduction. Surgical treatment of cancer of the oesophagus is associated with a high morbidity and mortality. Minimally invasive surgery has been proposed as an alternative to try to reduce these complications; however, at this time there are not many studies that evaluate the oncological validity of this method. The objective of this work is to give a preliminary audit of the results of our experience in both surgical techniques, with special emphasis on the oncopathological aspects (resection margins and lymph nodes).

Material and method. Between April 2003 and February 2007, 40 patients diagnosed with distal oesophageal cancer were surgically intervened at Charing Cross Hospital, London, 24 open and 16 by laparoscopy in accordance with the surgeon responsible. Of these, $50 \%$ received neoadjuvant chemotherapy. Both groups were homogeneous for age, sex, ASA, tumour 
stage and tumour location. In all cases, the pathological tumour stage (TNM), the tumour distal margin, tumour proximal margin, tumour circumference and number of resected lymph nodes, were collected in a data base.

Results. The number of resected lymph nodes was similar in both groups; (19 for open and 18 for laparoscopy). The mean distal tumour margin for the group treated by open surgery was $4.9 \mathrm{~cm}$ compared to 4.3 in the group treated by laparoscopy $(p=0.578)$. The mean proximal tumour margin for the group treated by open surgery was $8.4 \mathrm{~cm}$ compared to $4.6 \mathrm{~cm}$ in the laparoscopy group $(p=0.004)$ and tumour circumference margin was positive in 11 patients (45\%) belonging to the open group compared to 5 patients $(33 \%)$ in the laparoscopy group $(p=0.519)$.

Conclusions. In our experience, laparoscopic surgery for cancer of the oesophagus appears to show similar initial results to those of open surgery as regards the number of resected lymph nodes and resection margins.

Key words: Cancer of the oesophagus. Minimally invasive surgery. Transhiatal oesophagectomy.

\section{Introducción}

En la actualidad el cáncer de esófago es el tercero más frecuente de los cánceres gastrointestinales y el séptimo en la lista de los cánceres en el mundo occidental ${ }^{1}$. La incidencia de este cáncer está aumentando en los últimos años a expensas fundamentalmente de aquellos con localización en la unión gastroesofágica. El pronóstico de esta patología es pobre, en la mayoría de los casos estos enfermos precisan tratamiento multidisciplinario entre oncólogos, radioterapeutas y cirujanos. Clásicamente estos tumores localizados en el tercio inferior y en la unión gastroesofágica se han abordado por vía transtorácica con linfadenectomía extensa, lo que aumenta el riesgo de morbilidad temprana en favor de una mejor supervivencia a largo plazo ${ }^{2,3}$ o por vía transhiatal, según técnica de Orringer, con menor morbilidad pero asumiendo una teórica peor supervivencia a largo plazo ${ }^{4}$. En los estudios comparativos parece que en general se encuentra menor morbilidad para la vía transhiatal, sin grandes diferencias estadísticas en términos de supervivencia libre de enfermedad y supervivencia general para los tumores de la unión gastroesofágica y sí una mejor supervivencia a largo plazo para los adenocarcinomas de tercio distal5,6.

La cirugía sigue siendo el único tratamiento curativo para estos enfermos ${ }^{7}$, si bien, gracias a los nuevos protocolos de neoadyuvancia, se han descrito mejores resultados cuando se comparan con los obtenidos con la cirugía solamente 8,9 . La discusión en la actualidad se centra en las nuevas técnicas quirúrgicas con abordaje mínimamente invasivo y los protocolos de neoadyuvancia.

Varios estudios han comparado los resultados de la esofagectomía transhiatal abierta con la laparoscópica en el cáncer de esófago en términos de morbilidad postoperatoria ${ }^{10-13}$, y las ventajas del abordaje laparoscópico son significativas en cuanto a menor pérdida sanguínea, menor estancia hospitalaria y más temprana recuperación postoperatoria. Sin embargo, los resultados oncológicos todavía no se han valorado adecuadamente en un estudio aleatorizado, y son excepcionales los trabajos que abordan este tema ${ }^{12,14}$. El propósito de este trabajo es auditar nuestros resultados iniciales con la esofagectomía asistida por laparoscopia teniendo como referencia nuestros estándares de cirugía transhiatal abierta, y con especial atención a los márgenes de resección y ganglios linfáticos.

\section{Material y métodos}

Entre abril de 2003 y enero de 2007, se intervino, en el Hospital Charing Cross de Londres, a 40 pacientes diagnosticados de cáncer de esófago distal y de la unión gastroesofágica (20 Siewert tipo I y 20 Siewert tipo II) ${ }^{15}$; se realizó a todos ellos una esofagectomía transhiatal; 24 por vía abierta (ATH) y 16 por vía laparoscópica (LTH). La asignación a uno u otro grupo dependió del criterio del cirujano responsable del paciente; todas las intervenciones las realizó el mismo equipo quirúrgico. El $50 \%$ de los pacientes intervenidos según LTH recibieron tratamiento quimioterápico neoadyuvante frente al $62 \%$ de los intervenidos según ATH, que se aplica en nuestro centro a todos los pacientes con un estadio tumoral superior a T2NO. Ambos grupos fueron homogéneos para edad, sexo, ASA y estadio tumoral (tabla 1).

En todos los casos se recogió de modo prospectivo en una base de datos el estadio tumoral patológico (TNM) de acuerdo con la sexta edición de la American Joint Committee of Cancer (AJCC) ${ }^{16}$, el margen distal tumoral (MDT), el margen proximal tumoral (MPT), el margen circunferencial tumoral (MCT) y el número de ganglios linfáticos resecados. Estas mediciones se tomaron según el informe del patólogo una vez fijada la pieza en formol y de acuerdo con las guías del Real Colegio de Patólogos de Gran Bretaña, en las que se considera afectación positiva la presencia de células tumorales a menos de $1 \mathrm{~mm}$ del margen circunferencial en el estudio histológico ${ }^{17}$.

El análisis estadístico se realizó con la versión del programa SPSS 14.0 para Windows. Los valores de p para la comparación entre grupos

TABLA 1. Características de los pacientes

\begin{tabular}{lccc}
\hline & LTH $\mathbf{( n = 1 6 )}$ & ATH $\mathbf{( n = 2 4 )}$ & $\mathbf{p}$ \\
\hline Edad, media \pm DE & $66,5 \pm 13,08$ & $66,7 \pm 12,4$ & 0,956 \\
Sexo, varón/mujer & $12 / 4$ & $20 / 4$ & \\
ASA & 3 & 5 & 0,305 \\
I & 9 & 12 & \\
II & 3 & 6 & \\
IIV & 1 & 1 & \\
Quimioterapia & $50 \%$ & $62 \%$ & \\
Estadio tumoral & & & \\
T0 & 2 & 3 & \\
T1 & 2 & 5 & \\
T2 & 3 & 14 & \\
T3 & 7 & 1 & \\
T4 & 2 & 66 & \\
N+ (\%) & 53 & & \\
Localizacion tumoral & & 13 & \\
Siewert I & 7 & 11 & \\
Siewert II & 9 & $17 / 6$ & \\
Histología & & & \\
Adenocarcinoma/ & $11 / 3$ & & \\
$\quad$ escamoso & & & \\
Barrett/displasia & 2 & & \\
$\quad$ alto grado & &
\end{tabular}

ASA: American Society of Anesthesiologist; ATH: transhiatal abierta; DE: desviación estándar; LTH: transhiatal laparoscópica. 
Valentí V et al. Esofagectomía transhiatal por vía abierta y vía laparoscópica para el cáncer de esófago: análisis de los márgenes de resección y ganglios linfáticos

se calcularon aplicando la prueba de la t de Student. Para los valores no numéricos se utilizó el test de la $\chi^{2}$ de Pearson. Los parámetros con distribución normal se presentan como media y desviación estándar.

\section{Técnica quirúrgica}

La técnica utilizada, tanto para el abordaje abierto como el laparoscópico, es la descrita por Orringer et al ${ }^{4}$. Para el abordaje laparoscópico se procede con el paciente en decúbito supino y con el cuello extendido para exposición del lado izquierdo. Con el cirujano entre las piernas, la intervención se inicia con el tiempo abdominal a través de 5 trocares supraumbilicales insertando a través de uno de ellos un separador de Natahson ${ }^{\circledR}$.

El estómago se moviliza a lo largo de la curvatura mayor y los vasos cortos siguiendo la dirección de distal a proximal, preservando en la disección proximal el origen de la arteria gastroepiploica derecha. Los vasos gástricos izquierdos se seccionan y la linfadenectomía del tronco celíaco se extiende hasta el pilar derecho del diafragma. Se practicó una linfadenectomía D2 a excepción del grupo 10 en todos los casos según clasificación de Akiyama ${ }^{18}$. Una vez disecada la unión esofagogástrica seccionamos la vena frénica izquierda que permite dividir el diafragma y ensanchar el hiato para realizar la disección transmediastínica del esófago inferior con resección de dos parches pleurales (límite lateral de la disección). Los otros límites de la resección son la aorta (límite posterior) y el pericardio (límite anterior). La disección se extiende proximalmente hasta la vena pulmonar. Una vez se la ha alcanzado se da por terminado el tiempo abdominal y se procede a realizar la disección cervical y sección esofágica.

Por medio de una sonda nasogástrica que se ha exteriorizado previamente a través de la unión gastroesofágica se lleva un fleboextractor hasta el cuello y fijado al extremo distal del esófago se uiliza para el stripping del tercio superior del esófago. Al mismo tiempo, una minilaparotomía de $7 \mathrm{~cm}$, aproximadamente, se extiende desde el trocar supraumbilical en dirección craneal para la colocación de un HandPort ${ }^{\circledR}$ por el que se exterioriza el espécimen y con la ayuda de una GIA se construye un tubo gástrico de $3-4 \mathrm{~cm}$ de diámetro a expensas de la curvatura mayor. En ese momento el neumoperitoneo se restablece y el tubo gástrico, suturado a la sonda nasogástrica, asciende bajo visión directa hasta el cuello. La intervención se completa con una anastomosis esofagogástrica laterolateral y la colocación de 2 drenajes torácicos en la cavidad pleural desde el campo abdominal bajo visión laparoscópica exteriorizados por los orificios de 2 trocares.

\section{Resultados}

En la tabla 2 se recogen las características de los pacientes según tipo de intervención para el número de ganglios resecados, MDT, MPT y MCT. El número de ganglios linfáticos resecados fue casi idéntico en ambos grupos. Se obtuvieron menos de 15 ganglios en la pieza quirúrgica en 4 pacientes tratados por LTH (8-33) y en 8 por ATH (4-39). El MDT medio para el grupo tratado con ATH fue similar al obtenido en el grupo tratado con LTH. Sin embargo, el MPT medio para el grupo tratado con ATH fue significativamente superior al tratado con LTH. Asimismo, hubo una mayor afectación del MCT en el grupo tratado con ATH, sin diferencias significativas.

En ningún caso se convirtió la vía laparoscópica a la vía abierta. No hubo mortalidad postoperatoria.

TABLA 2. Características de los tumores

\begin{tabular}{lccc}
\hline & LTH $(\mathbf{n}=\mathbf{1 6})$ & ATH $(\mathbf{n}=\mathbf{2 4})$ & $\mathbf{p}$ \\
\hline MPT & $4,6 \pm 1,6$ & $8,4 \pm 3,6$ & 0,004 \\
MDT & $4,3 \pm 2,2$ & $4,9 \pm 2,8$ & 0,578 \\
MCT (+/) & $5 / 11$ & $11 / 13$ & 0,519 \\
Ganglios aislados & $18 \pm 8,3$ & $19 \pm 10,6$ & 0,597 \\
\hline
\end{tabular}

ATH: transhiatal abierta; LTH: transhiatal laparoscópica; MCT: margen circunferencial tumoral; MDT: margen distal tumoral; MPT: margen proximal tumoral.

Los datos se presentan como media \pm desviación estándar.

\section{Discusión}

El tratamiento quirúrgico del cáncer de esófago distal y de la unión gastroesofágica es en la actualidad la única posibilidad de curación para estos enfermos ${ }^{7}$. La esofagectomía transhiatal, al evitar la toracotomía, presenta en general un índice de complicaciones postoperatorias pulmonares menor cuando se compara con los procedimientos transtorácicos ${ }^{19-21}$. En un intento ulterior de disminuir la morbilidad de estos pacientes y mejorar la visión de la disección mediastínica del esófago se han ido desarrollando diferentes abordajes laparoscópicos. La principal ventaja de la esofagectomía laparoscópica transhiatal es la mejor visualización de las estructuras mediastínicas relacionadas con el tumor hasta el nivel de la bifurcación traqueal sin necesidad de usar el retractor. De esta manera, esta operación minimiza la posible inestabilidad hemodinámica frecuentemente encontrada por el uso del retractor y disección manual en la movilización esofágica ${ }^{12}$.

Puesto que la esofagectomía es el tratamiento fundamental para los pacientes diagnosticados de cáncer de esófago localmente avanzado, los objetivos del tratamiento quirúrgico deben ser conseguir en la intervención adecuados márgenes quirúrgicos y apropiadas linfadenectomías. La laparoscopia, por tanto, debe ser capaz de reproducir los resultados oncológicos de los abordajes clásicos abiertos.

La extensión longitudinal por la submucosa esofágica es una característica común a todos los tipos de carcinomas de esófago, por lo que se ha considerado idealmente que los márgenes proximal y distal tumorales para la resección en el cáncer de esófago deben ser de 10 y 5 $\mathrm{cm}$, respectivamente, una vez resecada la pieza y en su estado natural sin sufrir regresión por el formol ${ }^{22}$. Recientemente, un trabajo del grupo del Memorial ${ }^{23}$ ha demostrado que márgenes de resección superiores a $3,8 \mathrm{~cm}$, con la pieza fijada en formol, o de $5 \mathrm{~cm}$, con la pieza in situ, se asocian a un mejor pronóstico para los tumores de la unión gastroesofágica tratados únicamente con cirugía, siempre que se realice una cirugía $\mathrm{R} 0$ y se incluyan al menos 15 ganglios en la pieza quirúrgica. En nuestro trabajo, en ningún paciente el MPT y el MDT estaban afectados por el tumor y se consiguió preservar estos márgenes en las piezas fijadas en formol, tanto para la vía abierta como la laparoscópica, si bien en cerca del $50 \%$ de nuestros pacientes se aplicó tratamiento neoadyuvante. Hay que destacar que en el grupo laparoscópico (LTH) había 2 casos con diagnóstico de displasia de alto grado sobre esófago de Barrett de extensa longitud, por lo que el MPT en este grupo fue menor cuando se comparó con el grupo tratado con cirugía abierta $(\mathrm{ATH})$, con diferencias estadísticamente significativas para este valor. En todos los casos la sección esofágica se realizó a nivel cervical dejando un muñón esofágico proximal de unos $3 \mathrm{~cm}$.

La extensión de la resección circunferencial se ha correlacionado con el pronóstico postoperatorio de estos enfermos, con una supervivencia peor a 2 años para aquellos con margen positivo ${ }^{17}$. Nuestro trabajo muestra una tendencia para la afectación positiva en la vía abierta frente a la laparoscópica (el $45 \%$ con ATH frente al $33 \%$ 
con LTH), sin diferencias estadísticamente significativas debido al tamaño de la muestra $(p=0,519)$. Sin embargo, en los pacientes con diagnóstico de carcinoma avanzado es aconsejable realizar una resección parcial de los pilares del diafragma y de las pleuras parietales locales para lograr una resección oncológica completa ${ }^{21}$.

La linfadenectomía en el cáncer de esófago sigue siendo un aspecto controvertido. Los objetivos de la linfadenectomía se deben centrar en lograr un número de ganglios suficientes para poder establecer correctamente el estadio de la enfermedad y en reducir el riesgo de recurrencia locorregional. De esta manera se incrementa el porcentaje de pacientes con resecciones $\mathrm{R} 0$, pues el ratio de ganglios afectados frente al total de ganglios resecados tiene significación pronóstica ${ }^{24}$. En este sentido, con la aplicación de las técnicas laparoscópicas se ha de lograr resecar el mismo número de ganglios que mediante el abordaje laparotómico. Nuestros resultados muestran un porcentaje medio de ganglios resecados similar para ambos grupos (19 para ATH frente a 18 para la LTH) y en consonancia con los criterios aprobados por la ISDE en 1995, que recomienda un mínimo de 15 ganglios en la pieza quirúrgica para poder establecer correctamente el estadio patológico tumoral ${ }^{25}$.

Este trabajo no tiene por objetivos establecer conclusiones acerca de los posibles factores pronósticos asociados a la recurrencia de la enfermedad, puesto que para ello, sin duda, sería necesario realizar un estudio con una muestra más amplia y un seguimiento a largo plazo mayor; sin embargo, parece mostrar la posibilidad de abordar estos tumores de forma segura por vía laparoscópica en términos de radicalidad oncológica.

Pensamos que aunque la vía abierta sigue siendo la referencia para el abordaje de estos tumores, el abordaje laparoscópico transhiatal está ganando evidencia como alternativa válida en los tumores localmente avanzados de la unión esofagogástrica y tercio inferior de esófago.

\section{Bibliografía}

1. Parkin DM, Bray F, Ferlay J, et al. Global Cancer Statistics, 2002. CA Cancer J Clin. 2005;55:74-108.

2. Lewis I. The surgical treatment of carcinoma of the oesophagus with special reference to new operation for growths of middle third. Br J Surg. 1946;34:18-31.

3. Tanner IC. The present position of carcinoma of the oesophagus. Postgrad Med J. 1947;23:109-39.

4. Orringer MB, Marshall B, Stiding MC. Transhiatal esophagectomy for benign and malignant disease. J Thorac Cardiovasc Surg. 1993; 105:256-77.

5. Hulscher JB, van Sandwick JW, De Boer AG, et al. Extended transthoracic resection compared with limited transhiatal resection for adenocarcinoma of the esophagus. N Engl J Med. 2002;347:1662-9.
6. Hagen JA, Peters JH, DeMeester TR. Superiority of extendeden bloc esophagogastrectomy for carcinoma of the lower esophagusand cardia. J Thorac Cardiovasc Surg. 1993;106:850-8.

7. Allum WH, Griffin SM, Watson A, et al. Guidelines for the management of oesophageal and gastric cancer. Gut. 2002;50 Suppl V:v123.

8. Walsh N, Noonan N, Hollywood D, Kelly A, Keeling N, Hennessy TP. A comparison of mutimodal herapy and surgery for esophageal adenocarcinoma. N Engl J Med. 1996;335:462-7.

9. Benecewicz J, Clark P, Smith D, et al. Surgical resection with or without preoperative chemotherapy in oesophageal cancer: a randomized controlled trial. Lancet. 2002;9319:1727-33.

10. Avital S, Zundel N, Szomstein S, Rosenthal R. Laparoscopic transhiatal esophagectomy for esophageal cancer. Am J Surg. 2005;190:69-74.

11. Bernabe KQ, Bolton JS, Richardson WS. Laparoscopic hand-assisted versus open transhiatal esophagectomy: a case-control study. Surg Endosc. 2005;19:334-7.

12. Van den Broek WT, Makay O, Berends FJ, Yuan JZ, Houdijk AP, Meijer S, et al. Laparoscopically assisted transhiatal resection for malignancies of the distal esophagus. Surg Endosc. 2004;18:812-7.

13. Bann S, Moorthy K, Shaul T, Foley R. Laparoscopic transhiatal surgery of the eesophagus. JSLS. 2005;9:376-81.

14. Azagra JS, Goergen M, Lens V, Ibanez-Aguirre JF, Schiltz M, et al. Present state of mini-invasive surgery (MIS) in esophageal and gastric cancer. Clin Transl Oncol. 2006;8:139-41.

15. Siewert JR, Feith M, Stein HJ. Biological and clinical variations of adenocarcinoma at the esophago-gastric junction: relevance of a topographic-anatomic subclassification. J Surg Oncol. 2005;90:13946.

16. Greene FL, Page DL, Fleming ID, et al. AJCC Cancer Staging Manual. 6.a ed. New York: Springer-Verlag; 2002.

17. Mapstone NP. Minimum data set for esophageal carcinoma histopathology reports. Royal College of Pathologists. 1998. Disponible en: http://www.rcpath.org/resouces/pdf oesphagaelcarcinoma

18. Akiyama H. Japanese Classification of Gastric Carcinoma. Japanese Research Society for Gastric Cancer. 1. ${ }^{\mathrm{a}}$ ed. Tokyo: Kanehara \& Co; 1995.

19. Goldfadem D, Orringer MB, Appelman HD, Kalish R. Adenocarcinoma of distal esophagus and gastric cardia: comparison of results of transhiatal esophagectomy and thoracoabdominal esophagogastrectomy. J Thorac Cardiovascular Surg. 1986;91:242-7.

20. Millikan KW, Silverstein J, Hart V, et al. A 15 year review of esophagectomy for carcinoma of the esophagus and cardia. Arch Surg. 1995;130:617-24.

21. Lee RB, Maller JI. Esophagectomy for cancer. Surg Clin North Am. 1997;77:1169-95.

22. Allum WH, Griffin SM, Watson A, Collin-Jones D. Association of Upper Gastrointestinal Surgeons of Great Britain and Ireland, the British Society of Gastroenterology and the British Association of Surgical Oncology. Guidelines for the management of oesophageal and gastric cancer. Gut. 2002;50:V1-23.

23. Barbour AP, Rizk NP, Gonen M, Tang L, Bains MS, Rusch VW, et al. Adenocarcinoma of the gastroesopgaheal junction: influence of esophageal resection margin and operative approach on outcome. Ann Surg. 2007;246:1-8.

24. Roder JD, Bush R, Stein JH, et al. Ratio of invaded to removed lymph nodes as a predictor of survival in squamous cell carcinoma of the oesophagus. Br J Surg. 1994;81:410-3.

25. Fumagalli U. Resective surgery for cancer of the toracic esophagus. Results of a consensus conference held at the Vlth World Congress of the International Society for Diseases of the Esophagus. Dis Esophagus. 1996;9:30-8. 